\title{
Filaria specific antibody response profiling in plasma from anti-retroviral naïve Loa loa microfilaraemic HIV-1 infected people
}

Ghislain Donald Njambe Priso ${ }^{1,2}$, Abel Lissom ${ }^{1,2}$, Loveline N. NGU1,4, Nadesh N. Nji ${ }^{1}$, Jules Colince Tchadji ${ }^{1,2}$, Thibau Flaurant Tchouangueu 1,5, Georgia E. Ambada ${ }^{1,2}$, Carole Stéphanie Sake Ngane ${ }^{1,3,4}$, Brigitte Laure Dafeu ${ }^{1,2}$, Larissa Djukouo ${ }^{1,4}$, Inès Nyebe ${ }^{1,3}$, Suzanne Magagoum ${ }^{1,2}$, Apeh Alfred Ngoh ${ }^{1,6}$, Ouambo Fotso Herve ${ }^{1,8}$, Rosario Garcia ${ }^{7}$, Anna Gutiérrez ${ }^{7}$, Arinze S. Okoli ${ }^{12}$, Charles O. Esimone ${ }^{13}$, Flobert Njiokou ${ }^{2}$, Chae Gyu Park ${ }^{11}$, Alain Bopda Waffo, ${ }^{9,0}$ and Godwin W. Nchinda ${ }^{1 *}$

\begin{abstract}
Background: In West and Central Africa areas of endemic Loa loa infections overlap with regions of high prevalence of human immunodeficiency virus type 1 (HIV-1) infections. Because individuals in this region are exposed to filarial parasites from birth, most HIV-1 infected individuals invariably also have a history of filarial parasite infection. Since HIV1 infection both depletes immune system and maintains it in perpetual inflammation, this can hamper Loa loa filarial parasite mediated immune modulation, leading to enhanced loaisis.
\end{abstract}

Methods: In this study we have assessed in plasma from asymptomatic anti-retroviral (ARV) naive Loa loa microfilaraemic HIV-1 infected people the filarial antibody responses specific to a filariasis composite antigen consisting of Wbgp29-BmR1BmM14-WbSXP.

The antibody responses specific to the filariasis composite antigen was determined by enzyme linked immunosorbent assay (ELISA) in plasma from ARV naïve Loa loa microfilaraemic HIV-1 infected participants. In addition the filarial antigen specific lgG antibody subclass profiles were also determined for both HIV-1 positive and negative people.

Results: Both Loa loa microfilaraemic HIV-1 positive and negative individuals showed significantly higher plasma levels of $\operatorname{lgG1}(P<0.0001)$, IgG2 $(P<0.0001)$ and $\lg M(P<0.0001)$ relative to amicrofilaraemic participants. A significant increase in IgE $(P<0.0001)$ was observed exclusively in Loa loa microfilaraemic HIV-1 infected people. In contrast there was a significant reduction in the level of $\operatorname{lgG} 4(p<0.0001)$ and $\operatorname{lgG} 3(P<0.0001)$ in Loa loa microfilaraemic HIV-1 infected individuals.

Conclusions: Loa loa microfilaraemia in ARV naïve HIV-1 infected people through differential reduction of plasma levels of filarial antigen specific lgG3, IgG4 and a significant increase in plasma levels of filarial antigen specific lgE could diminish Loa loa mediated immune-regulation. This in effect can result to increase loaisis mediated immunopathology in antiretroviral naive HIV-1 infected people.

Keywords: HIV-1, Loa loa, Microfilaraemia, Loaisis, African eye worm

* Correspondence: nsehleseh@gmail.com

'Laboratory of vaccinology/biobanking, CIRCB, Messa, Yaounde, Cameroon

Full list of author information is available at the end of the article

(c) The Author(s). 2018 Open Access This article is distributed under the terms of the Creative Commons Attribution 4.0 International License (http://creativecommons.org/licenses/by/4.0/), which permits unrestricted use, distribution, and reproduction in any medium, provided you give appropriate credit to the original author(s) and the source, provide a link to the Creative Commons license, and indicate if changes were made. The Creative Commons Public Domain Dedication waiver (http://creativecommons.org/publicdomain/zero/1.0/) applies to the data made available in this article, unless otherwise stated. 


\section{Background}

Loa Loa, or the African eye worm, is a human filarial parasite endemic in West and Central Africa, with over 13 million people currently infected with the parasite [1, 2]. During L. loa infection (Loiasis) the adult filarial parasite can live in subcutaneous tissues for up 17 years [3] and is capable of producing microfilariae (MF) in peripheral blood which are the further transmitted by the dipteran vector (chrysops spp). The great majority of people infected with Loa loa show little or no visible symptoms $[4,5]$. This is because $L$. loa, like all helminth infections, modulates immune responses to reach a safe parasite-host equilibrium, where either high levels of loa loa microfilaria (microfilaraemic loiasis) or no apparent microfilaria (amicrofilaraemic loiasis) can be found in peripheral blood $[6,7]$. Some reports have suggested that elevated levels of filarial parasite antigen specific IgG4 antibody subclass is essential for asymptomatic infection [8]. But other reports have suggested that increased expression of nonspecific polyclonal IgE [9] and elevated levels of specific IgG4 [10] have been associated with loaisis. Nevertheless all antibody isotypes, including IgM, IgE and IgG, are known to play a major role in mediating protection to filarial infections [11].

In sub-Saharan Africa, areas of intense endemic helminth infections overlap with regions of high prevalence of HIV-1 [12]. Therefore the risk of concomitant infection with filarial parasites and HIV-1 is high. In regions of high $L$. lao endemicity like Central and West Africa with concurrent high HIV-1 prevalence, few studies have looked at the interaction between these two infections. Both L. loa and HIV-1 are chronic infections where the pathogen can persist for longer periods in the absence of sterilizing immunity. HIV-1 infection depletes and maintains the immune system in a sustained inflammatory state, rendering the host more susceptible to other infectious diseases. In contrast filarial parasites in the majority of cases modulate the host's immune response to ensure their survival, thereby maintaining asymptomatic infection with elevated plasma levels of parasite antigen specific IgG4 [10]. This is generally associated with a generalized state of hypo-responsiveness which ensures long term filarial parasite survival [11].

In this study, we have investigated the profile of filarial antigen specific antibody responses of Loa loa microfilaraemic ARV naïve HIV-1 infected people in comparison with HIV-1 negative participants. This is because filarial parasites employ both filarial antigen specific and nonspecific immunomodulatory strategies to escape elimination from the human host. However ARV naïve HIV-1 infection depletes the immune system and with concomitant persistent loa loa microfilaraemia the profile of filarial antigen specific antibody responses is not known. The immune depletion and sustained inflammation associated with HIV-1 infection might alter immune modulation and change the profile of filarial antigen specific antibody responses in the context of microfilaraemic loaisis. Thererfore, the profiles of filarial antigen specific antibody responses in Loa loa microfilaraemic HIV-1 infected people could reveal possible interactions between these two infections.

Using a mixture of four recombinant filarial parasite antigens, consisting of Wbgp29, BmR1, BmM14 and WbSXP, we have assessed antibody responses in both microfilaraemic and amicrofilaraemic long standing ARV naïve HIV-1 infected people in the CIRCB AFRODEC cohort [13]. Antibody assays based on Bm14, WbSXP, Wbgp29 or BmR1 have previously been demonstrated to show excellent sensitivity $(>90 \%)$ in the detection of filarial specific antibodies $[14,15]$. Bm14 and WbSXP, for example, are highly reactive with their respective antibodies in plasma from microfilaraemic individuals. More so prior studies have reported the Bm14 antibody test as a sensitive marker for infection or heavy exposure to filarial parasites $[14,16,17]$. This implies that this mixture of recombinant filarial antigen is suitable for assessing Loa loa specific antibody responses.

The consequence of ARV naïve HIV-1 infection was a significant increase in plasma levels of filariasis composite antigen specific IgE. This was in association with a significant decrease in serum levels of filarial antigen specific IgG4 and IgG3, clearly suggesting a reduction in Loa loa mediated immunomodulation.

\section{Methods}

\section{Study population}

Participants were 21 to 65 years old and samples were collected as part of the CIRCB AFRODEC study. The CIRCB AFRODEC (African HIV-1 dendritic cell targeted vaccine) cohort study was conducted in Cameroon from November 2012 to November 2016 and enrolled antiretroviral therapy (ARV) naïve HIV-1 infected people. During the course of 4 years 199 participants out of the 766 members of CIRCB AFRODEC cohort who repeatedly complained of filarial infection were recruited for this study. In addition confirmed 99 HIV-1 negative participants complaining for microfilarae infecions were also monitored alongside their HIV-1 positive counterparts as controls. Finally 25 Loa loa microfilaraemic antiretroviral therapy (ARV) naïve HIV-1 infected participants who remained Loa loa positive during the course of 4 years were included in the analysis. 25 amicrofilaraemic ARV therapy naïve HIV-1 infected individuals with no signs of loaisis were also included. Similarly a control group of HIV-1 negative individuals was separated into 25 Loa loa microfilaraemic and amicrofilaraemic participants. In addition to people who did not provide consent, participants who had been diagnosed 
with Hepatitis B virus, Hepatitis C virus, Dengue virus, Mycobacterium tuberculosis, or malaria were excluded from the study. Absolute numbers of helper $\mathrm{CD}^{+} \mathrm{T}$ cells for HIV-1 positive participants was determined in fresh whole blood by BD multitest CD3/CD8/CD45/CD4 and TruCount tubes (BD Biosciences, San Jose, USA) according to the manufacturer's instructions. Plasmatic HIV-1 viral load was determined on the m2000rt machine using the Abbott Real-Time HIV-1 Assay protocol.

\section{Study design (Fig. 1)}

Members of the CIRCB AFRODEC cohort who are either HIV-1 positive or negative, were included based on the presence or absence of Loa loa microfilaraemia during a period of 4 years. Recruited microfilaraemic participants are those who showed at least two microfilaraemia per year during the period of the study. Amicrofilaraemic participants were Loa loa negative throughout the 4 years of the study.

\section{Sample collection and processing}

Blood was collected into 4-ml plastic vacuum blood spray-coated K2EDTA tubes (Vacutestkirma, Italy). All samples were stored at room temperature and processed within $4 \mathrm{~h}$ of collection. To obtain plasma, samples were centrifuged at $2000 \mathrm{rpm}$ for $10 \mathrm{~min}$ at $4{ }^{\circ} \mathrm{C}$. The plasma fraction was harvested in sterile conditions under the hood, aliquoted in small, single-use volumes and stored at $-20{ }^{\circ} \mathrm{C}$ until use. All plasma obtained from antiretroviral naive $\mathrm{HIV}-1$ infected participants was heat inactivated for $30 \mathrm{~min}$ at $56{ }^{\circ} \mathrm{C}$ prior to ELISA assay.

\section{Filarial diagnosis}

Parasitological examination of individuals was done by microscopic examination of $10 \%$ Giemsa stained peripheral blood $(10 \mu \mathrm{l})$, both as thin and thick films. In the CIRCB AFRODEC cohort screening for Loa loa microfilaraemia was performed three times annually for each participant. Three independent confirmations of Loa loa infection by different researchers were necessary for a sample to be considered positive.

\section{Enzyme linked immunosorbent assay (ELISA) for IgM, IgG and $\lg \mathrm{E}$}

Filariasis composite antigen Wbgp29-BmR1-BmM14WbSXP was diluted in PBS and $100 \mu$ l containing $100 \mathrm{ng} /$ well were added to high binding 96-well flat bottom microsorp (Thermo Fisher Scientific) ELISA plates and incubated overnight at $4{ }^{\circ} \mathrm{C}$. The following day, plates were washed three times with PBST (PBS with 0 . 05\% Tween-20) and blocked either with 3\% BSA or 1x Roti block (Carl ROTH, Karlsruhe, Germany) for 1 hour at $37^{\circ} \mathrm{C}$. After an additional washing step, $100 \mu \mathrm{l} /$ well of plasma diluted (1:500) in PBS was added into

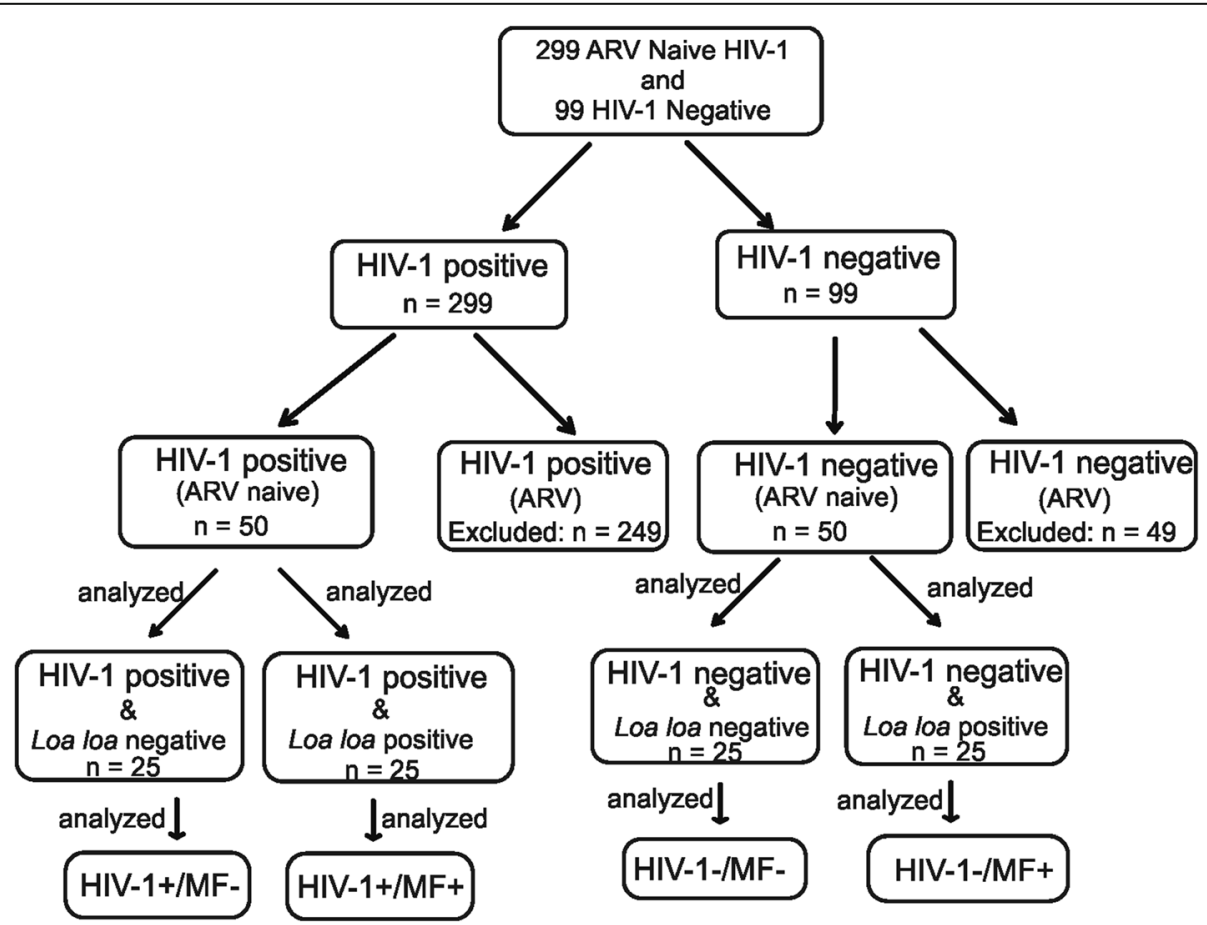

Fig. 1 Study flow displaying screening and recruitment of study participants. Members of the CIRCB AFRODEC cohort which are either HIV-1 positive or negative were included based on the presence or absence of Loa loa microfilaraemia during a period of 4 years. Recruited microfilaraemic participants are those showed at least two microfilaraemia per year during the period of the study 
corresponding wells in triplicates and incubated for 2 hours at $37^{\circ} \mathrm{C}$.

Next the plates are washed five times as described above. Then $100 \mu \mathrm{l}$ horseradish peroxidase (HRP)-conjugated anti-human IgG (1:2000) and horseradish peroxidase (HRP)-conjugated anti-human IgG1, IgG2, IgG3, IgG4, or IgM (1:4000) antibodies were added, and the plates incubated for $1 \mathrm{~h}$ at $37^{\circ} \mathrm{C}$. Similarly for the detection IgE, HRP-conjugated anti-human IgE diluted 1:1000 was used. Antibody isotypes and subclasses used in this study were mouse anti-human IgG Fc (clone JDC-10), mouse anti-human IgM (clone UHB), mouse antihuman IgE Fc (clone B3102E8), mouse anti-human IgG1 Fc (clone HP6001), mouse anti-human IgG2 Fc (clone 31-7-4), mouse anti-human IgG3 Hinge (clone HP6050) and mouse anti-human IgG4 pFc' (clone HP6023), all HRP conjugate all procured from Southern Biotech ${ }^{\circ}$ (Birmingham, USA). Plates were then washed five times and $100 \mu \mathrm{l}$ of 2,2'-Azino-bis (3-ethylbenzthiazoline-6sulfonic acid) or ABTS one Component HRP substrate was added to each well and incubated in the dark for $30 \mathrm{~min}$. The enzyme substrate reaction was stopped by adding a stop solution (Southern Biotech, Birmingham,USA). The optical densities (OD) at $405 \mathrm{~nm}$ were read using a multiscan Fc Elisa microplate reader (Thermoscientific, USA). All plasma was tested in triplicate and the mean OD values were determined.

\section{Statistics}

Statistical analyses were done using the software GraphPad PRISM version 6.0 (Graphpad Software). Comparisons between groups were by the nonparametric Mann-Whitney-U test (for paired data), Student's t-test (for unpaired data) and Kruskal-Wallis test. Viral load was expressed on a $\log 10$ scale. $P$ values of 0.05 or less were considered significant.

\section{Results}

\section{Study population}

199 ARV naïve HIV-1 infected individuals from the CIRCB AFRODEC cohort and 99 HIV-1 negative people who repeatedly complained of filarial infection were monitored for 4 years. During 4 years eligible participants were separated into 4 groups based on their HIV-1 status and persistent Loa loa microfilaraemia. Excluded during the course of the study were individuals diagnosed occasionally with Loa loa microfilaraemia, Hepatitis B virus, Hepatitis C virus, Dengue virus, Mycobacterium tuberculosis and malaria. Participants retained for both HIV-1 positive and negative groups based on persistent Loa loa microfilaraemia or amicrofilaraemia were analyzed. Amongst the HIV-1 infected individuals, 25 were HIV-1 positive without microfilaraemia (HIV+/MF-) and 25 were Loa loa microfilaraemic $(\mathrm{HIV}+/ \mathrm{MF}+)$. As shown in Table 1 for the HIV + MF-, $10(40 \%)$ were males and 15 (60\%) were females. Regarding HIV + MF+ participants 09 (36\%) were males and 16 (64\%) were females respectively. In addition 25 microfilaraemia negative (HIV-/MF-) and 25 microfilaraemic (HIV-/MF+) HIV-1 negative participants who were repeatedly tested as part of the CIRCB AFRODEC cohort were analysed as controls. The age distribution between the four different groups was similar (Table 1).

As shown in Table 1, microfilaria negative HIV-1 infected participants differed significantly $(p<0.05)$ in their median helper $\mathrm{CD}^{+} \mathrm{T}$ cell counts from microfilaraemic HIV-1 infected people (compare 499 with 367.5 cells $/ \mathrm{mm}^{3}$ ). In addition plasma viral load in Loa loa microfilaraemic HIV-1 infected individuals was significantly higher $(P<0.05)$ than in microfilaraemia negative HIV-1 infected participants.

Loa loa microfilaraemia results in enhanced plasma levels of Filariasis composite antigen specific IgM in both HIV-1 negative and positive individuals

When plasma levels of filariasis composite antigen specific IgM were considered both Loa loa microfilaraemic HIV-1 negative (in Fig 2a compare $\mathrm{HIV}^{-} / \mathrm{MF}^{-}$with $\mathrm{HIV}^{-} / \mathrm{MF}^{+}$) and positive (Fig $2 \mathrm{~b}$ compare $\mathrm{HIV}^{+} / \mathrm{MF}^{-}$ with $\mathrm{HIV}^{+} / \mathrm{MF}^{+}$) individuals showed significantly higher plasma levels $(P<0.001)$ vis a vis microfilaraemia negative individuals (Fig 2 a $\&$ b). Loa loa microfilaraemia in both HIV-1 positive and negative individuals was associated with significantly elevated OD values of filariasis composite antigen specific IgM implying a continuous exposure to filarial antigens. The OD

Table 1 Study population characteristics

\begin{tabular}{|c|c|c|c|c|c|c|}
\hline \multirow{2}{*}{$\begin{array}{l}\text { Variable } \\
\text { Gender }\end{array}$} & \multirow{2}{*}{$\begin{array}{l}\mathrm{HIV}^{-} / \mathrm{MF}^{-}(n=25) \\
\text { Male and Female }\end{array}$} & \multirow{2}{*}{$\begin{array}{l}\mathrm{HIV}^{-} / \mathrm{MF}^{+}(\mathrm{n}=25) \\
\text { Male and Female }\end{array}$} & \multicolumn{2}{|c|}{$\mathrm{HIV}^{+} / \mathrm{MF}^{-}(\mathrm{n}=25)$} & \multicolumn{2}{|c|}{$\mathrm{HIV}^{+} / \mathrm{MF}^{+}(\mathrm{n}=25)$} \\
\hline & & & Male & Female & Male & Female \\
\hline Participants (\%) & $8(32) 17(68)$ & $11(44) 14(56)$ & $10(40)$ & $15(60)$ & $9(36)$ & $16(64)$ \\
\hline Median Age (IQR) & $34(21-41)$ & $49.50(45.25-55.25)$ & $34(32-43.50)$ & $37(27-46)$ & $38(28-46)$ & $35.5(28.75-45)$ \\
\hline Mf count (MF/ml) Median & N/A & $1800(10-4800)$ & \multicolumn{2}{|l|}{ N/A } & \multicolumn{2}{|c|}{$986.7(1-3200)$} \\
\hline Median CD4 counts (cells/mm³) & N/A & N/A & \multicolumn{2}{|c|}{499 (IQR 310.5-886.8) } & \multicolumn{2}{|c|}{$367.5\left(\right.$ IQR 157.8-460.8) ${ }^{*}$} \\
\hline Median Viral load (Log 10 copies/ml) & N/A & N/A & \multicolumn{2}{|c|}{3.05 (IQR 1.76-3.85) } & \multicolumn{2}{|c|}{$5.04(\mathrm{IQR} 4.530-5.5)^{*}$} \\
\hline
\end{tabular}

${ }^{*} \mathrm{P}<0.05, \mathrm{~N} / \mathrm{A}=$ Not Applicable, IQR $=$ interquartile range, $\mathrm{ns}=$ not significant, $\mathrm{MF}^{+}=$microfilaria positive 


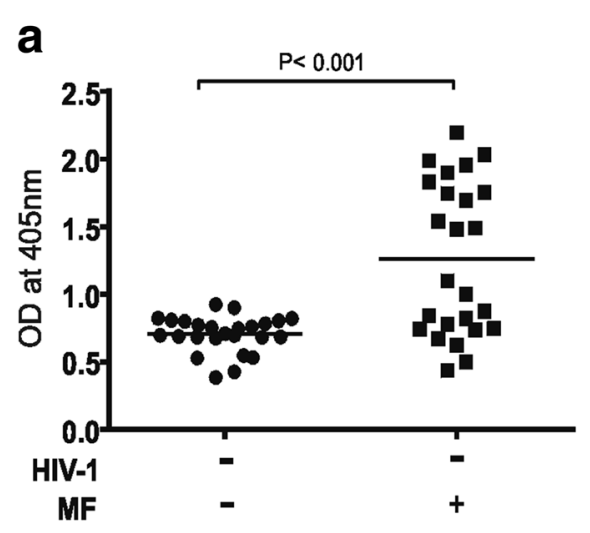

b

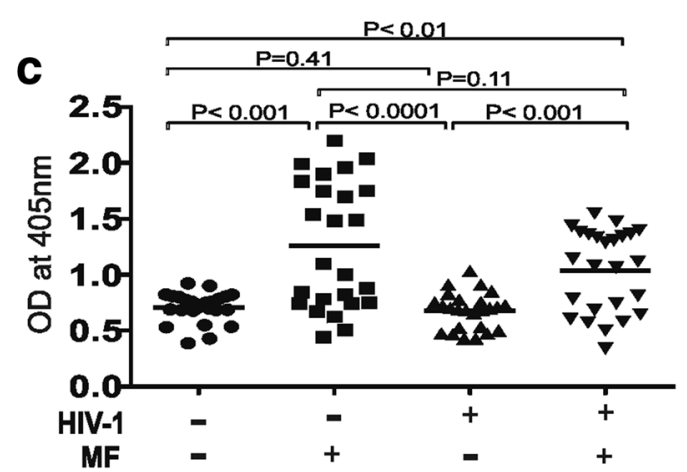

Fig. 2 Plasma levels of Filariasis composite antigen specific IgM in Loa loa microfilaraemia positive and negative ARV naïve HIV-1 infected people compared to seronegative people. As shown in (a) \& (b) loa loa microfilaraemia results in significantly $(P<0.001)$ higher plasma levels of IgM in both HIV-1 negative and positive individuals (compare $\mathrm{HIV}^{\top} / \mathrm{MF}^{+}$with $\mathrm{HIV}^{+} / \mathrm{MF}^{+}$). In (c) a comparative statistically analysis is made between the four groups with respect to plasma levels of their Filariasis composite antigen specific $\operatorname{lgM}$. In (c) a slight but insignificant reduction $(P<0.115)$ is observed in plasma levels of antigen specific IgM in Loa loa microfilaraemic ARV naïve HIV infected people. Statistical analysis was done with prism graph path 6.0, unpaired t test, Mann-Whitney-U tests and Kruskal-Wallis test

values showed a slight negative correlation with the microfilaria burden ( $r=-0.084$ data not shown). In Fig 2c a statistical analysis is shown for the four different groups with respect to plasma levels of filariasis composite antigen specific IgM. When HIV-1 positive and negative individuals were compared, except for a slight reduction in the plasma level of antigen specific IgM of Loa loa microfilaraemic ARV naïve HIV-1 infected people there was no significant difference in plasma level of filariasis antigen specific IgM in both microfilaraemia HIV-1 positive and negative individuals (Fig 2c). Thus elevated plasma levels of filarial antigen specific IgM are associated with Loa loa microfilaraemia in both HIV-1 negative and positive people.

ARV naïve HIV-1 infections results in elevated plasma levels of filariasis composite antigen specific lgE exclusively in Loa loa microfilaraemic individuals

A unique situation was observed with plasma levels of filariasis composite antigen specific IgE where significantly $(P<0.0001)$ elevated plasma levels of antigen specific IgE was observed exclusively for Loa loa microfilaraemic ARV naïve HIV-1 infected people. In contrast little or no antigen specific IgE was detected in the other three groups (compare Fig. 3 a\&b). In Fig. 3c a statistical analysis is shown for the four different groups with respect to plasma levels of filariasis antigen specific IgE. Thus ARV naïve HIV-1 infection escalates plasma levels of filariasis antigen specific IgE, which could result in an increase in immunopathology.

\section{Filariasis composite antigen specific lgG antibody} responses in Loa loa microfilaraemic antiretroviral naïve HIV-1 infected individuals

With respect to plasma levels of filariasis composite antigen specific IgG; Loa loa microfilaraemic HIV-1 negative individuals showed significantly higher levels $(P$ $<0.01$ ) relative to amicrofilaraemia individuals (in Fig. 4a compare $\mathrm{HIV}^{-} / \mathrm{MF}^{-}$with $\mathrm{HIV}^{-} / \mathrm{MF}^{+}$). Plasma levels of filariasis composite antigen specific IgG in ARV naïve HIV-1 infected participants in contrast did not differ significantly between Loa loa microfilaraemia positive and negative individuals (Fig. 4b compare $\mathrm{HIV}^{+} / \mathrm{MF}^{-}$with $\mathrm{HIV}^{+} / \mathrm{MF}^{+}$).

In Fig 4c a statistical analysis is shown for the four different groups with respect to plasma levels of filariasis 


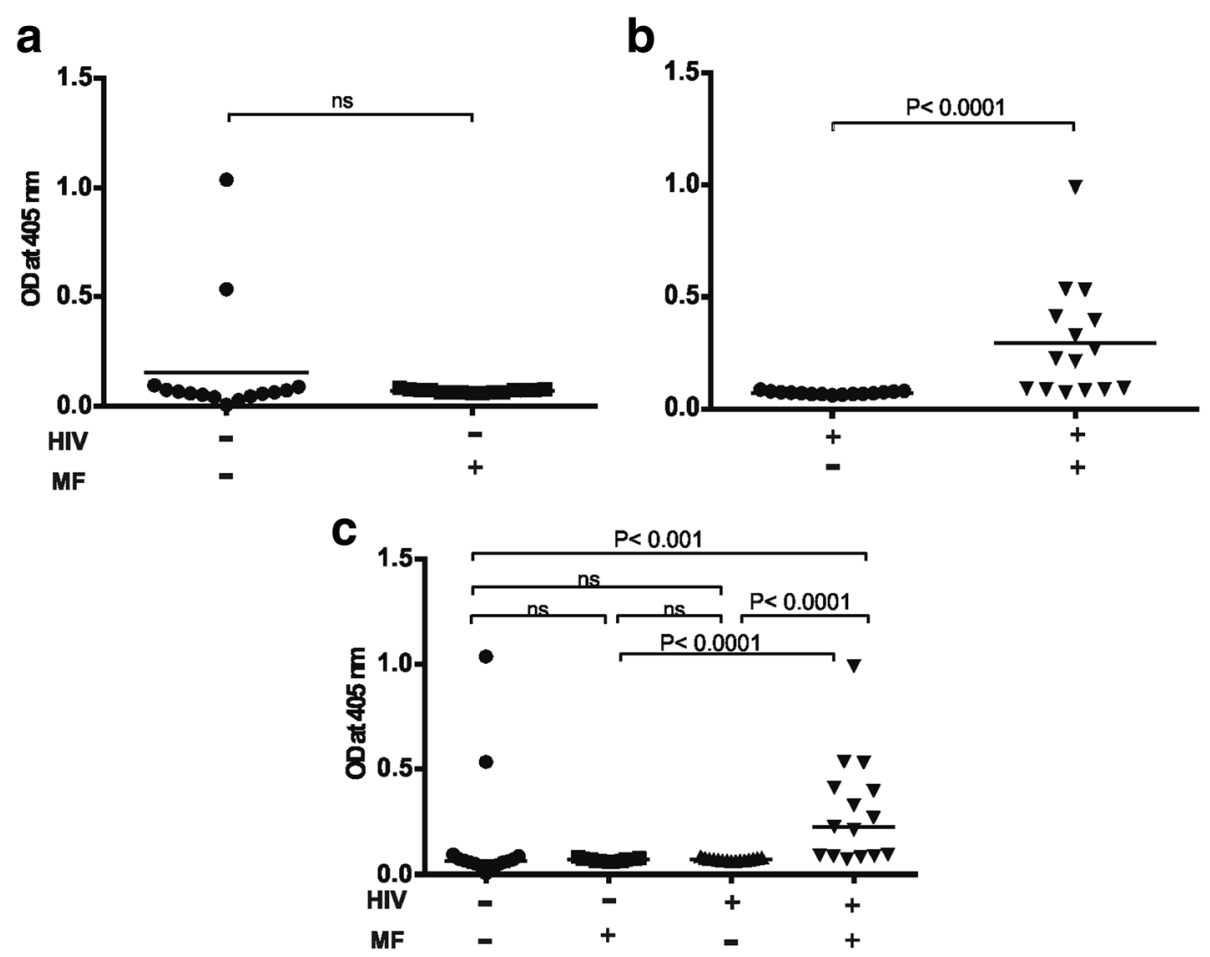

Fig. 3 Plasma levels of Filariasis composite antigen specific lgE in Loa loa microfilaraemia positive and negative ARV naïve HIV-1 infected people compared to seronegative people. As shown in (a) \& (b) Loa loa microfilaraemia results in significantly $(P<0.001)$ higher plasma levels of antigen specific lgE exclusively in ARV naïve HIV-1 infection (compare $\mathrm{HIV}^{-} / \mathrm{MF}^{+}$with $\mathrm{HIV}^{+} / \mathrm{MF}^{+}$). In (c) a comparative statistically analysis is made between the four groups with respect to plasma levels of their Filariasis composite antigen specific IgE. In (c) a significantly higher $(P<0.0001)$ plasma levels of antigen specific lgE is observed exclusively in Loa loa microfilaraemic ARV naïve HIV infected people. Statistical analysis was done with prism graph path 6.0, unpaired t test, Mann-Whitney-U test and Kruskal-Wallis test

composite antigen specific IgG. During this study ARV naïve HIV-1 infected people generally demonstrated significantly higher plasma levels $(P<0.001)$ of filariasis composite antigen specific IgG relative to HIV negative individuals irrespective of Loa loa microfilaraemia. In this case when microfilaraemia negative seronegative individuals are compared with ARV naïve HIV-1 infected people a significantly higher plasma level of filariasis antigen specific IgG was observed for the HIV-1 infected people (Fig. 4c). Increased filariasis specific IgG levels in ARV naïve HIV-1 infected people could be an indication of the absence of effective immunodulation which is relevant in the long term survival of microfilaria. This in effect could escalate filarial parasite mediated immunopathology.

Increased plasma levels of Filariasis composite antigen specific lgG1 and lgG2 antibody subclass responses in Loa loa microfilaraemic individuals

The plasma level of filariasis composite antigen specific IgG1 was significantly $(P<0.0001)$ elevated during Loa loa microfilaraemia in both HIV-1 positive and negative individuals (Fig. 5a\&b). In Fig. 5c a statistical analysis is shown for the four different groups with respect to plasma levels of filariasis composite antigen specific IgG1. When participants were compared with respect to HIV-1 status a significant increase in plasma level of IgG1 was observed in microfilaraemia negative ARV naïve HIV-1 infected individuals relative to seronegative individuals. In contrast when microfilaraemic HIV-1 positive and negative individuals were compared a significant reduction $(P<0.05)$ in IgG1 was observed in ARV naïve HIV-1 infected participants (Fig. 5c). This implies that Loa loa microfilaraemic ARV naïve HIV-1 infection can lead to a significant reduction in plasma levels of filariasis composite antigen specific IgG1.Similarly Loa loa microfilaraemia resulted in elevated plasma levels of IgG2 in both HIV-1 positive and negative individuals (Fig. $5 \mathrm{~d} \& \mathrm{e}$ ). When HIV-1 infected people were compared with their negative counter parts no significant difference $(P<0.2)$ was observed between microfilaraemic HIV-1 positive and negative people with respect to plasma levels of filariasis composite antigen specific IgG2 (Fig. 5f).

Reduction in plasma levels of Filariasis composite antigen specific IgG3 and lgG4 antibody subclass responses in Loa loa microfilaraemic ARV naïve HIV-1 infected individuals Plasma level of filariasis composite antigen specific IgG3 was significantly $(P<0.0001)$ higher in Loa loa 

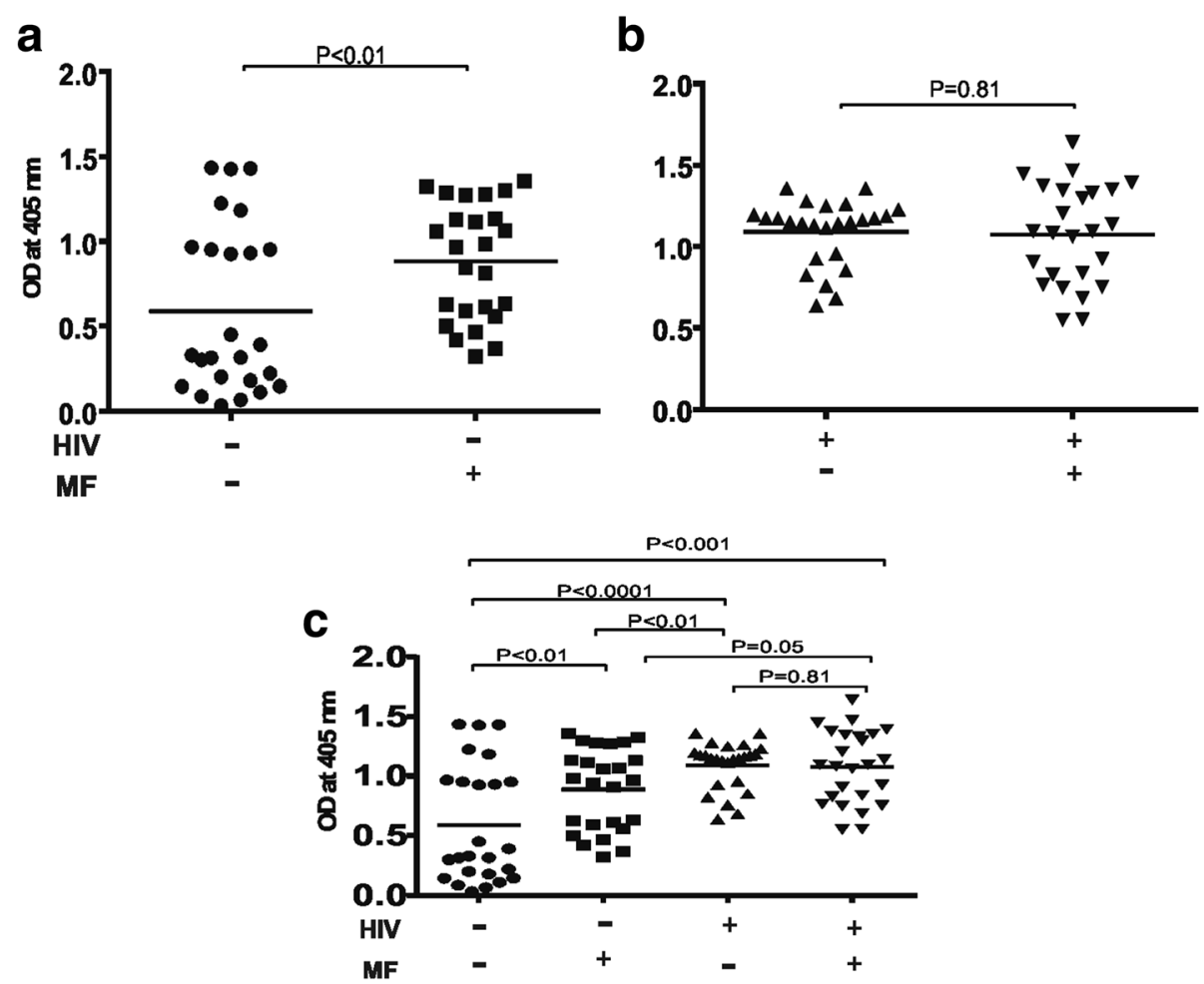

Fig. 4 Plasma levels of Filariasis composite antigen specific lgG in Loa loa microfilaraemia positive and negative ARV naïve HIV-1 infected people compared to seronegative people. As shown in $(\mathbf{a})$ \& (b) Loa loa microfilaraemia results in significantly $(\mathrm{P}<0.001)$ higher plasma levels of antigen specific lgG exclusively in HIV-1 negative individuals (compare HIV-/MF- with HIV-/MF+). In (c) a comparative statistically analysis is made between the four groups with respect to plasma levels of their Filariasis composite antigen specific lgG. Statistical analysis was done with prism graph path 6.0, unpaired t test Mann-Whitney-U tests and Kruskal-Wallis test

microfilaraemic HIV-1 negative individuals (Fig. 6a). In contrast in ARV naïve HIV-1 infected people there was a significant reduction in plasma levels of filariasis composite antigen specific IgG3 (Fig. 6b). In Fig. 6c a statistical analysis is shown for the four different groups with respect to plasma levels of filariasis composite antigen specific IgG3. When participants were next compared with respect to HIV-1 status a significant reduction in plasma level of IgG3 was associated with microfilaraemic ARV naïve HIV-1 infection. This was in contrast to microfilaraemia negative individuals where significantly high plasma levels of filariasis composite antigen specific IgG3 was observed in ARV naïve HIV-1 infected individuals (Fig. 6c). On the other hand filariasis composite antigen specific IgG4 Plasma levels were reduced significantly $(\mathrm{P}<0.0001)$ in Loa loa microfilaraemic HIV-1 infected individuals compared to microfilaraemia negative HIV-1 positive people. In addition microfilaraemia negative HIV-1 infected people showed comparatively similar filariasis composite antigen specific IgG4 Plasma levels like Loa loa microfilaraemic HIV-1 negative individuals. (Fig. 6 d\&e). In Fig. $6 \mathrm{f}$ a statistical analysis is shown for the four different groups with respect to plasma levels of antigen specific IgG4. Comparing HIV-1 negative with positive individuals filariasis composite antigen specific IgG4 Plasma levels were generally higher in ARV naïve HIV-1 positive than negative.

\section{Discussion}

In this study we have assessed the impact of ARV naïve HIV-1 infection on the patterns of filariasis composite antigen specific antibody responses in microfilaremic loaisis by analyzing plasma profiles of filariasis composite antigen specific IgG, IgM and IgE amongst HIV-1 positive and negative participants. This is mainly because filairial antigen specific IgG, IgE and IgM have been demonstrated to play critical roles in acquired immunity to filarial parasite infection [18]. However in the context of microfilaraemia in ARV naïve HIV-1 infection where there is not only HIV-1 driven immune depletion but equally sustained inflammation little is known about the profiles of filarial antigen specific antibody responses. The need for this study becomes pertinent especially with recent reports indicating that the hitherto relatively less pathogenic loaisis $[4,5]$ can be a significant cause of mortality in Loa loa endemic countries [19]. Two main groups consisting of ARV naïve HIV-1 infected members of the CIRCB AFRODEC cohort and HIV-1 negative 


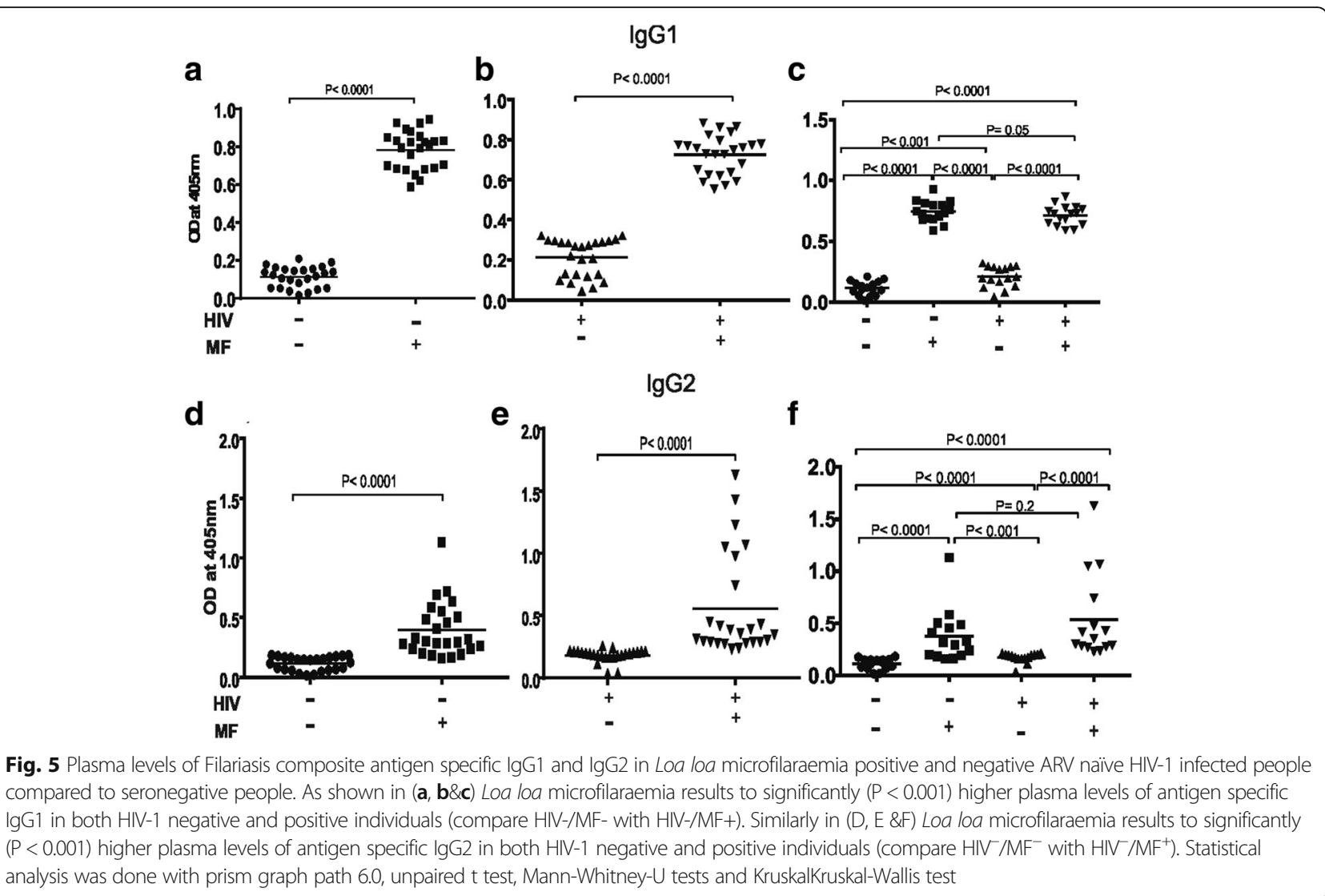

participants were used for this study. The ARV naïve HIV-1 infected group was further split into Loa loa microfilaraemia positive $\left(\mathrm{HIV}^{+} \mathrm{MF}^{+}\right)$and negative $\left(\mathrm{HIV}^{+} \mathrm{MF}^{-}\right)$ individuals respectively. Similarly the HIV-1 negative group consisted of microfilaraemia $\left(\mathrm{HIV}^{-} \mathrm{MF}^{+}\right)$positive and negative $\left(\mathrm{HIV}^{-} \mathrm{MF}^{-}\right)$individuals. All participants were members of the CIRCB AFRODEC cohort for the last 4 years and were repeatedly monitored for Loa loa microfilaraemia during this period.

Using a filariasis composite antigen consisting of Wbgp29-BmR1-BmM14-WbSXP in ELISA assay differences in filarial antigen specific antibody responses between the four subgroups was analyzed based on the presence or absence of microfilariae. Our study revealed significantly elevated plasma levels of filariasis composite antigen specific IgG1, IgG2 and IgM in plasma from both microfilaraemic ARV naïve HIV-1 positive and negative participants. These findings agree with previous studies which linked elevated levels of filarial antigen specific IgG1 and IgG2 with the presence of microfilariae [20]. The HIV-1 infection status had a significant effect on the plasma level of filariasis composite antigen specific IgE, IgG3 and IgG4 in Loa loa microfilaraemic individuals. Thus, while in HIV-1 negative people Loa loa microfilaraemia resulted to a significant increase in plasma levels of filarial antigen specific IgM, IgG, IgG1, IgG2,
IgG3 and IgG4, it produced a completely different outcome in ARV naïve HIV-1 infected people. In this case apart from IgG1 and IgG2 plasma levels of filariasis composite antigen specific IgG3 and IgG4 were significantly decreased in microfilaraemic ARV naïve HIV-1 infection. In addition microfilaraemic ARV naïve HIV-1 infected people also showed an exclusive increased in plasma levels of filarial antigen specific IgE which was significantly higher than all the groups described in this study.

Microfilaraemia during lymphatic filariasis has been linked to elevated levels of species specific IgG4 [21, 22]. Previous studies have shown that Loa loa specific IgG4 was elevated in both microfilaraemic and amicrofilaraemic persons [9, 10, 23]. However using the filariasis composite antigen assay we detected significantly higher plasma levels of antigen specific IgG4 in microfilaraemic HIV-1 negative people relative to their HIV-1 positive counterparts. This agrees with previous observation indicating that microfilariae were the main inducers of IgG4 [24]. In this context elevated levels of filarial antigen specific IgG4 have been incriminated in immunomodulation which is necessary for the filarial parasite to evade immune destruction [9, 25-27].

The significant reduction in plasma levels of IgG4 in Loa loa microfilaraemic HIV-1 infected people is probably due to immune depletion resulting from the ARV naïve HIV-1 

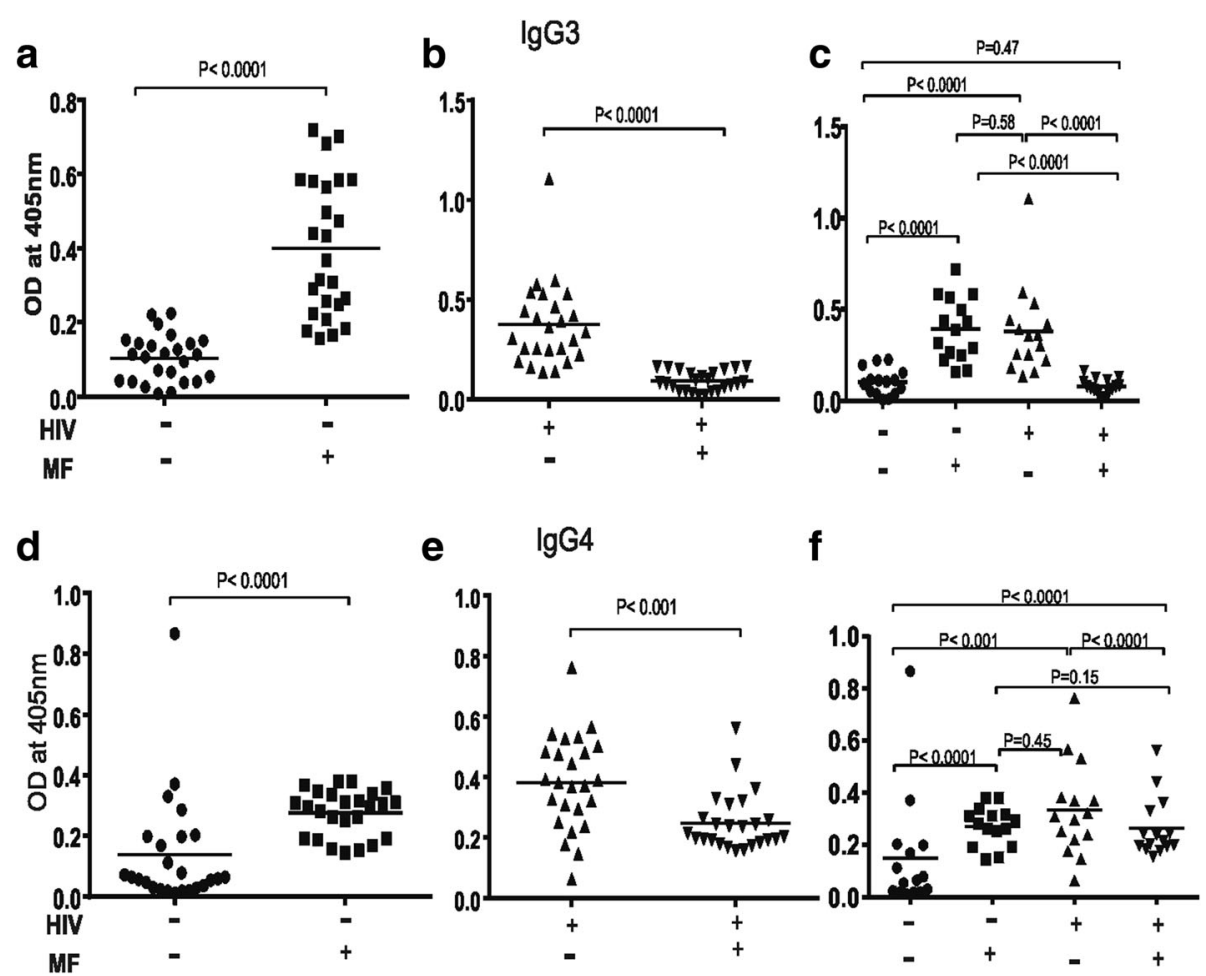

Fig. 6 Plasma levels of Filariasis composite antigen specific lgG3 and lgG4 in Loa loa microfilaraemia positive and negative ARV naïve HIV-1 infected people compared to seronegative people. As shown in (a) Loa loa microfilaraemia results to significantly $(P<0.0001)$ elevated plasma levels of antigen specific lgG3 in HIV-1 negative people on the one hand and a significant reduction $(\mathrm{P}<0.0001$ ) of antigen specific lgG3 in HIV-1 positive individuals on the other hand. Similarly in (e) Loa loa microfilaraemia results to significantly $(P<0.0001)$ higher plasma levels of antigen specific lgG4 in HIV-1 negative individuals in contrast to a significant reduction $(\mathrm{P}<0.001)$ in HIV-1 positive individuals (compare HIV-/MF- with HIV-/MF+). Here the effect of Loa loa microfilaraemic HIV-1 infection was a significant reduction in plasma levels of filariasis composite antigen specific lgG3 $(P<0.0001)$ and lgG4 $(P<0.001)$. Statistical analysis was done with prism graph path 6.0, unpaired t test, Mann-Whitney-U tests and Kruskal-Wallis test

infection. This implies that the reduction in plasma levels of filarial antigen specific IgG4 mediated by ARV naïve HIV-1 infection even in the presence of microfilariae could potentially exacerbate Loa loa filarial mediated immunopathology. This is especially so as the decrease in filarial antigen specific IgG4 was accompanied in microfilaraemic HIV-1 positive people by a significant increase in plasma levels of filarial antigen specific IgE. Several reports suggest that the rate of induction, magnitude and the ratio between, the IgE and IgG4 isotypes are major determinants of the clinical outcomes of lymphatic filariasis. In this way, patients with chronic lymphatic disease have been reported with elevated ratios of filarial antigen specific IgE to IgG4. This was in contrast to asymptomatic microfilaraemic patients who presented high plasma levels of IgG4 with a corresponding low IgE to IgG4 ratio. [22, 28-31]. This implies that in microflaraemic ARV naïve HIV-1 infected people the significantly high plasma levels of filarial antigen specific IgE accompanied with reduced specific IgG4 could be an indication of increased immunopathology. Compared to microfilaria negative HIV-1 positive individuals there was enhanced immune depletion in Loa loa microfilaraemic ARV naïve HIV-1 infected people. Here Loa loa microfilaraemic ARV naïve HIV-1 infection also resulted in a significant increase in plasma viral load. A two log increased in viral load relative to microfilaria negative participant is a clear indication that dual chronic Loa loa microfilaraemia and HIV-1 infection can escalate the expansion of the virus. Thus in depleting the immune system ARV naive HIV-1 infection can differentially influence antifilarial antigen specific IgE, Ig3 and IgG4 in microfilaraemic loaisis. On the other hand Loa loa microfilaraemia can escalate immune depletion in HIV-1 infection and significantly influence HIV-1 viral load. This bidirectional effect of Loa loa microfilaraemia and ARV naïve HIV-1 infection on each other can escalate morbidity and mortality.

\section{Conclusions}

Thus Loa loa microfilaraemia in ARV naïve HIV-1 infected people, through differential reduction of plasma levels of filarial antigen specific IgG3, IgG4 and a significant increase in plasma levels of filarial antigen specific IgE, could diminish Loa loa mediated immuneregulation. This can result in an increase in loaisis mediated immunopathology in antiretroviral naive HIV-1 infected people. 


\section{Abbreviations}

ARV: Antiretroviral; BSA: Albumin Serum Bovin; ELISA: Enzyme-Linked Immunosorbent Assay; HIV-1: Human Immunodeficiency virus type 1; HRP: Horseradish Peroxidase; Ig: Immunoglobulin; L. loa: Loa loa; MF: Microfilariae; OD: Optical Densities; PBS: Phosphate Buffered Saline

\section{Acknowledgements}

We thank all participants who consented to participate in this study. We would like to thank the personnel of CIRCB medical section (Unite Technique) for their help in collecting the blood samples.

\section{Funding}

This work was supported by a Third world academy of sciences (grant number, 12059RG/BIO/AF/AC_G); EDCTP (grant number, TA.2010.40200.016); Canada rising star grant (grant number, 30-11-2012-01-05-2014); African Korean collaborative grand (grant number, NRF-2013KIA3A1A09076155) and CIRCB (grand number PTA-2012-2017)

\section{Availability of data and materials}

Information regarding data referring to the CIRCB AFRODEC cohort used in this manuscript are part of the data bank of the Chantal Biya international Reference center for research on the prevention and management of HIV/ AIDS, it is not possible to obtain them by URL. The data are available upon request from the CIRCB administration for researchers who meet the criteria for access to confidential data. Access to data can be obtained through email nsehleseh@circb-cm or by phone number+237,222,315,456.

\section{Authors' contributions}

GWN, CGP, and ABW conceived the study; GWN,CGP, GDN and AL designed the study protocol; GDN, AL, LN, NN,JCT,TFT, GA, CSSN, BLD, LD, IN, SM,AAN, $\mathrm{OFH}, \mathrm{RG}$ and $\mathrm{AG}$ performed the experiments; GWN,GDN and AL drafted the manuscript; $A O, C E, F N, C G P, A B W$ and GWN critically revised the manuscript for intellectual content. All authors read and approved the final manuscript. GWN is the guarantor of the paper.

\section{Ethics approval and consent to participate}

All human samples in the AFRODEC Cohort were collected with written informed consent under clinical protocols approved by the Republic of Cameroon National Ethics Committee (protocol number 2014/10/499/CE/ CNERSH/SP) the CIRCB institutional review board (protocol number 14-11) and the Cameroon government administrative authorization (authorization number 631-1112). The AFRODEC cohort consists of adult HIV-1 infected participants aged 21 to 65 years who were naïve to antiretroviral therapy.

\section{Consent for publication}

Not applicable.

\section{Competing interests}

The authors declare that they have no competing interests.

\section{Publisher's Note}

Springer Nature remains neutral with regard to jurisdictional claims in published maps and institutional affiliations.

\footnotetext{
Author details

${ }^{1}$ Laboratory of vaccinology/biobanking, CIRCB, Messa, Yaounde, Cameroon. ${ }^{2}$ Department of animal biology and Phisiology, University of Yaounde I, Yaounde, Cameroon. ${ }^{3}$ Department of Microbiology, University of Yaounde I, Yaounde, Cameroon. ${ }^{4}$ Department of biochemistry, University of Yaounde I, Yaounde, Cameroon. ${ }^{5}$ Department of biochemistry, University of Dschang, Yaounde, Cameroon. ${ }^{6}$ Department of biomedical sciences, University of Dschang, Dschang, Cameroon. ${ }^{7}$ CSCB (Centre de santé catholique de Bikop), Bikop, Cameroon. ${ }^{8}$ Department of medical laboratory sciences, University of Buea, Buea, Cameroon. ${ }^{9}$ Department of Biological Sciences, College STEM, 1627 Hall Street, Montgomery, AL 36101, USA. ${ }^{10}$ Center for NanoBiotechnology Research, 1627 Harris Way, Montgomery, AL 36104, USA.

${ }^{11}$ Laboratory of Immunology, Brain Korea 21 PLUS Project for Medical Science, Severance Biomedical Science Institute, Yonsei University College of Medicine, Seoul 03722, Republic of Korea. ${ }^{12}$ Gen $\varnothing k$ - Centre for Biosafety, Tromsø, Norway. ${ }^{13}$ Department of Pharmaceutical Microbiology \& Biotechnology, Nnamdi Azikiwe University, Awka, Nigeria.
}

Received: 25 October 2017 Accepted: 26 March 2018

Published online: 04 April 2018

\section{References}

1. Drame PM, Meng Z, Bennuru S, Herrick JA, Veenstra TD, Nutman TB. Identification and validation of Loa loa microfilaria-specific biomarkers: a rational design approach using proteomics and novel immunoassays. MBio. 2016;7(1):e02132-15

2. Zoure HG, Wanji S, Noma M, Amazigo UV, Diggle PJ, Tekle AH, et al. The geographic distribution of Loa loa in Africa: results of large-scale implementation of the rapid assessment procedure for Loiasis (RAPLOA) PLoS Negl Trop Dis. 2011;5(6):e1210.

3. Cho HY, Lee YJ, Shin SY, Song HO, Ahn MH, Ryu JS. Subconjuctival Loa loa with Calabar swelling. J Korean Med Sci. 2008;23(4):731-3.

4. Metzger WG, Mordmuller B. Loa loa-does it deserve to be neglected? Lancet Infect Dis. 2014;14(4):353-7.

5. Pion SD, Filipe JA, Kamgno J, Gardon J, Basanez MG, Boussinesq M. Microfilarial distribution of Loa loa in the human host: population dynamics and epidemiological implications. Parasitology. 2006;133(Pt 1):101-9.

6. Van Hoegaerden M, Chabaud B, Akue JP, Ivanoff B. Filariasis due to Loa loa and Mansonella perstans: distribution in the region of Okondja, HautOgooue Province, Gabon, with parasitological and serological follow-up over one year. Trans R Soc Trop Med Hyg. 1987;81(3):441-6.

7. Akue JP, Devaney E, Wahl G, Moukana H. Expression of filarial-specific lgG subclasses under different transmission intensities in a region endemic for loiasis. The American journal of tropical medicine and hygiene. 2002:66(3):245-50.

8. Adjobimey T, Hoerauf A. Induction of immunoglobulin G4 in human filariasis: an indicator of immunoregulation. Ann Trop Med Parasitol. 2010;104(6):455-64.

9. Akue JP, Hommel M, Devaney E. Markers of Loa loa infection in permanent residents of a loiasis endemic area of Gabon. Trans $\mathrm{R}$ Soc Trop Med Hyg. 1996:90(2):115-8.

10. Akue JP, Egwang TG, Devaney E. High levels of parasite-specific lgG4 in the absence of microfilaremia in Loa loa infection. Tropical medicine and parasitology : official organ of Deutsche Tropenmedizinische Gesellschaft and of Deutsche Gesellschaft fur Technische Zusammenarbeit. 1994:45(3):246-8.

11. Babu S, Nutman TB. Immunology of lymphatic filariasis. Parasite Immunol. 2014:36(8):338-46

12. Hotez PJ, Kamath A. Neglected tropical diseases in sub-saharan Africa: review of their prevalence, distribution, and disease burden. PLoS Negl Trop Dis. 2009;3(8):e412

13. Ambada GN, Ntsama CE, Nji NN, Ngu LN, Carole SN, Lissom A, et al. Phenotypic characterization of regulatory $T$ cells from antiretroviral naive HIV-1 infected people. Immunology. 2017;151(4):405-16.

14. Lammie PJ, Weil G, Noordin R, Kaliraj P, Steel C, Goodman D, et al. Recombinant antigen-based antibody assays for the diagnosis and surveillance of lymphatic filariasis - a multicenter trial. Filaria journal. 2004;3(1):9.

15. Pandiaraja P, Arunkumar C, Hoti SL, Rao DN, Kaliraj P. Evaluation of synthetic peptides of WbSXP-1 for the diagnosis of human lymphatic filariasis. Diagn Microbiol Infect Dis. 2010;68(4):410-5

16. Rao KV, Eswaran M, Ravi V, Gnanasekhar B, Narayanan RB, Kaliraj P, et al. The Wuchereria bancrofti orthologue of Brugia malayi SXP1 and the diagnosis of bancroftian filariasis. Mol Biochem Parasitol. 2000;107(1):71-80.

17. Weil GJ, Ramzy RM, El Setouhy M, Kandil AM, Ahmed ES, Faris R. A longitudinal study of Bancroftian filariasis in the Nile Delta of Egypt: baseline data and one-year follow-up. The American journal of tropical medicine and hygiene. 1999;61(1):53-8

18. Sahu BR, Mohanty MC, Sahoo PK, Satapathy AK, Ravindran B. Protective immunity in human filariasis: a role for parasite-specific IgA responses. $J$ Infect Dis. 2008:198(3):434-43.

19. Chesnais CB, Takougang I, Paguele M, Pion SD, Boussinesq M. Excess mortality associated with loiasis: a retrospective population-based cohort study. Lancet Infect Dis. 2017;17(1):108-16.

20. Nicolas L, Langy S, Plichart C, Deparis X. Filarial antibody responses in Wuchereria bancrofti transmission area are related to parasitological but not clinical status. Parasite Immunol. 1999;21(2):73-80.

21. Kurniawan A, Yazdanbakhsh M, van Ree R, Aalberse R, Selkirk ME, Partono F, et al. Differential expression of IgE and IgG4 specific antibody responses in asymptomatic and chronic human filariasis. J Immunol. 1993;150(9):3941-50.

22. Kwan-Lim GE, Forsyth KP, Maizels RM. Filarial-specific lgG4 response correlates with active Wuchereria bancrofti infection. J Immunol. 1990; 145(12):4298-305 
23. Klion AD, Vijaykumar A, Oei T, Martin B, Nutman TB. Serum immunoglobulin G4 antibodies to the recombinant antigen, LI-SXP-1, are highly specific for Loa loa infection. J Infect Dis. 2003;187(1):128-33.

24. Shiny C, Krushna NS, Archana B, Farzana B, Narayanan RB. Serum antibody responses to Wolbachia surface protein in patients with human lymphatic filariasis. Microbiol Immunol. 2009;53(12):685-93.

25. Jiraamonnimit C, Wongkamchai S, Boitano J, Nochot H, Loymek S, Chujun S, et al. A cohort study on anti-filarial lgG4 and its assessment in good and uncertain MDA-compliant subjects in brugian filariasis endemic areas in southern Thailand. J Helminthol. 2009;83(4):351-60.

26. Noordin R, Shenoy RK, Rahman RA. Comparison of two lgG4 assay formats (ELISA and rapid dipstick test) for detection of brugian filariasis. The Southeast Asian journal of tropical medicine and public health. 2003;34(4):768-70.

27. van der Neut Kolfschoten M, Schuurman J, Losen M, Bleeker WK, MartinezMartinez P, Vermeulen E, et al. Anti-inflammatory activity of human lgG4 antibodies by dynamic fab arm exchange. Science. 2007;317(5844):1554-7.

28. Hussain R, Grogl M, Ottesen EA. IgG antibody subclasses in human filariasis. Differential subclass recognition of parasite antigens correlates with different clinical manifestations of infection. J Immunol. 1987;139(8):2794-8.

29. Hussain R, Ottesen EA. IgE responses in human filariasis. III. Specificities of lgE and IgG antibodies compared by immunoblot analysis. J Immunol. 1985;135(2):1415-20.

30. Hussain R, Poindexter RW, Ottesen EA. Control of allergic reactivity in human filariasis. Predominant localization of blocking antibody to the lgG4 subclass. J Immunol. 1992;148(9):2731-7.

31. Yazdanbakhsh M, Paxton WA, Kruize YC, Sartono E, Kurniawan A, van het Wout A, et al. T cell responsiveness correlates differentially with antibody isotype levels in clinical and asymptomatic filariasis. J Infect Dis. 1993;167(4):925-31.

\section{Submit your next manuscript to BioMed Central and we will help you at every step:}

- We accept pre-submission inquiries

- Our selector tool helps you to find the most relevant journal

- We provide round the clock customer support

- Convenient online submission

- Thorough peer review

- Inclusion in PubMed and all major indexing services

- Maximum visibility for your research

Submit your manuscript at www.biomedcentral.com/submit 